\title{
Design of Chang'e No.three Soft Landing Orbit Based on GA and Texture Analysis
}

\author{
Jiani Xing ${ }^{1, a}$ \\ ${ }^{1}$ North China Electric Power University, Baoding, Hebei, China \\ a1398xjn@sina.com
}

Keywords: soft landing, dynamic programming, genetic algorithm, texture analysis..

\begin{abstract}
This paper the reduction of Chang'e no.3 soft landing and obstacle avoidance phase are studied. And based on that, there is an orbital control scheme with the aim of consumption the least fuel. In this scheme, the dynamic programming model that aims at consuming the least fuel is set up for the main reduction stage. Combined with the genetic algorithm, we get the optimal control scheme. During the obstacle avoidance phase, we use the texture analysis to quickly identify the surface imaging and automatically look for a safe landing area in order to avoid obstacle.
\end{abstract}

\section{Introduction}

Chang'e no.3 was successfully launched 1:30 a.m. on December 2, 2013 and arrived at the moon's orbit on December 6. Before the soft landing, Chang'e no.3 continued to fly in the elliptical orbit of which the perilune is $15 \mathrm{~km}$ high and the apolune is $100 \mathrm{~km}$ high. During this period, Chang'e no.3 kept a stable flight posture and confirmed the landing sensors and landing data again, making the final preparations for the starting altitude, speed and time of the soft landing. The key point of making sure Chang'e no.3 achieve a soft landing on the reserved area on the moon when it was flying at a high speed is to design of landing orbit and control strategy.

The basic design requirements of Chang'e no.3loft landing orbit: the prepared landing orbit should be an elliptical orbit of which the perilune is $15 \mathrm{~km}$ high and the apolune is $100 \mathrm{~km}$ high; the landing orbit is far from the perilune to the landing point; its landing process is divided into 6 stages. Each stage should conform to the state of key point; consume as less fuel as possible during the period of soft landing. Considering that the main reduction stage consumes the most time and energy among the 6 stages, the moon image analysis in the obstacle avoidance phase is crucial to the choice of safe landing area. In this paper, we mainly make an optimal design for the control scheme of these 2 stages.

\section{Design of main reduction stage}

Analysis of landing. At reduction stage, Chang'e no.3went from $15 \mathrm{~km}$ to $3 \mathrm{~km}$ from lunar surface, and the velocity by $1.7 \mathrm{~km} / \mathrm{s}$ also descended into $57 \mathrm{~m} / \mathrm{s}$. During this period, sustainer motor provided brake force to make Chang'e No.3descend by parabola, while attitude motor adjusted direction of sustainer motor in accordance with navigation information.

Establishment of dynamic programming model. Establishment of a dynamic programming model[1]. In order to simplify the analysis, polar coordinates system, whose origin is selenocenter, is adopted to evaluate dynamics analysis of reduction stage, while ignoring some influence factor, such as the moon's gravity and non spherical item, lunisdar gravitational perturbation and so on. Through analyzing the force situation and movement of Chang'e no.3,a dynamic equation is gotten as follows. 


$$
\left\{\begin{array}{l}
\frac{d v_{r}}{d t}=-\frac{g}{r^{2}}+\frac{v_{\theta}^{2}}{r}+a \sin \beta \\
\frac{d v_{\theta}}{d t}=-\frac{v_{r} v_{\theta}}{r}+a \cos \beta \\
\frac{d r}{d t}=v_{r} \\
\frac{d \theta}{d t}=\frac{v_{\theta}}{r}
\end{array}\right.
$$

g refers to the moon's gravity constant; $r 、 \theta 、 v_{r}, v_{\theta}$ respectively are detector selenocentric distance, polar angle, normal velocity and transverse velocity; a refers to thrust acceleration; $a(t)=\frac{F}{M_{0}-\dot{m} \times t}$,F refers to motor thrust, whose amplitude ranges from $F_{\text {min }} \leq F \leq F_{\text {max }} ; M_{0}$ refers to detector riding quality at initial time on this stage; $m$ refers to consumption rate in unit time; $\beta$ refers to angle of thrust direction .

While satisfying the beginning and end velocity for this stage, we can obtain an objective function through an evaluation criterion. And the target of evaluation criterion is to make fuel consumption minimum as an optional control strategy.

$$
\min f=\int_{0}^{t_{f}} \dot{m} d t
$$

Variables normalization treatment. Because the state parameters of above-mentioned equation have large difference in magnitude, so it will damage significand of the result by using this equation. Thus, normalization treatment to variables and optimizing variables are necessary in order to improve the computational accuracy. The concretely operation is shown as follows:

$$
\bar{r}=\frac{r}{r_{r e f}}, \bar{m}=\frac{m}{m_{\text {ref }}}, \quad \bar{v}=\frac{v}{v_{\text {ref }}}, \bar{F}=\frac{F}{F_{\text {ref }}}, \bar{a}=\frac{\bar{F}}{\bar{m}}
$$

$\bar{r}, \bar{m}, \bar{v}, \bar{F}, \bar{a}$ respectively are correspondingly normalized variables. The reference value of pammeres is shown as follows:

$$
r_{\text {ref }}=R_{\text {moon }}, m_{\text {ref }}=M_{0}, v_{\text {ref }}=\sqrt{\frac{g}{r_{r e f}}}, F_{r e f}=\frac{m_{r e f} \times v_{r e f}^{2}}{r_{r e f}}
$$

Acquisition optimization by GA. Genetic algorithm[2] is a random search algorithm, using the reference of natural selection and genetics theory in living nature. It can be applied to deal with the complex optimization problems, which the traditional search algorithm can not solve. It starts to search from the random initial value solution and reach a new solution through selection, intersection, mutation and chooses the individuals who have better fitness. After several evolution, algorithm converges to the best chromosome.

Based on GA,the calculation and analysis result of model is shown at part 3.

\section{Design for speedily obstacle avoidance system}

Principle of texture analysis. Texture analysis is a main and useful area of study in pattern recognition. Through luminance difference, thresholding size of colorship image and calculating rough regions of identifying image, this method can recognition obstacles quickly.

Utilizing texture analysis, through recognizing formation of lunar surface that is obtained at obstacle avoidance phase by detector, it's feasible to design an obstacle avoidance project.

Caculation of neighbourhood gray-level co-ocurrence matrix. we takes digital elevation model,which is shot by prober at $2.4 \mathrm{~km}$ as an example. First, Matlab is utilized to transform $2300 \mathrm{x}$ 2300 elevation map into 2300 x 2300 GLCM; then, 2300 x 2300 GLCM is converted into $92 \times 92$ GLCM that is referred as A, with making neighbourhood at $25 \times 25$. And the element $r_{i j}$ of each neighbourhood matrix is replaced by texture coarseness $\mathrm{R}$ that locates where pixel is. 
Segmentation of image texture. Setting threshold [3]referred as $\delta$, neighbourhood GLCM needs to threshold segment, then shown as follows:

$$
\left\{\begin{array}{l}
a_{i j}=0, a_{i j} \geq \delta \\
a_{i \bar{j}}=1, a_{i j}<\delta
\end{array}\right.
$$

So neighbourhood GLCM is transformed into matrix that only involves 0,1.Then,a picture is drawn by matlab after threshold segmenting(as shown in Figure 1.By this way, another digital elevation model,which is shot at $100 \mathrm{~m}$,is also transformed into a picture, whose neighbourhood is $50 \times 50$.

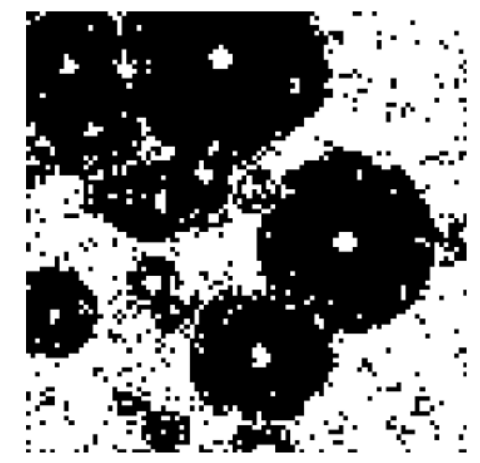

(a) $=10$

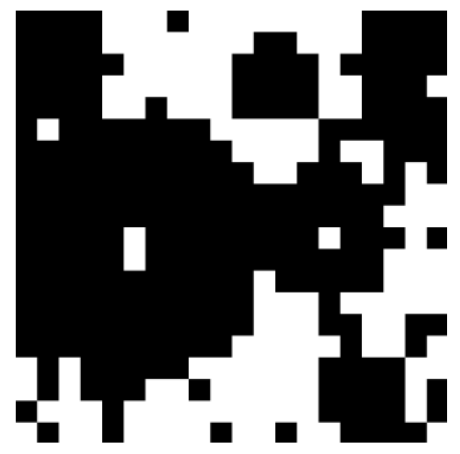

(b) $=30$

Figure 1 segmentation of digital elevation model texture

\section{Recognition of safe landing area autonomously}

We hypothesize detector's projection on lunar surface is at a neighbourhood of center in the image, what's more, if pixel value of this neighbourhood is 255(it's a white pixel), this area can be served as a safe landing area; if not, it can't stop to searching surrounding neighbourhood until the nearest white pixel region is discovered as a safe landing area. Then ,detector keeps close to it by adjusting attitude.

\section{Result of calculation and analysis}

While utilizing GA to execute global search the objective function at the dynamic programming model during main reduction stage, there are fractional parameters of algorithm: optimized parameters $\mathrm{n}$ is 6,population size $\mathrm{N}$ is 50,stochastic universal sampling method is adapted as a selection strategy, intersect probability $\mathrm{Pc}$ is 0.9 ,mutate probability $\mathrm{Pm}$ is 0.05 , iteration algebra $\mathrm{T}$ is 300.Finally,we can obtain a curves of distance of the moon as time measured(as shown in Figure 1), and a chart for consuming time and fuel(as shown in chart 1).During obstacle avoidance phase, texture analysis is used to recognize lunar surface speedily, when attitude engine works autonomously to adjust position. It's viable to get fuel consumption and time of Chang'e no.3 at this phase by matlab programming. The exact result is shown in Table 1. 


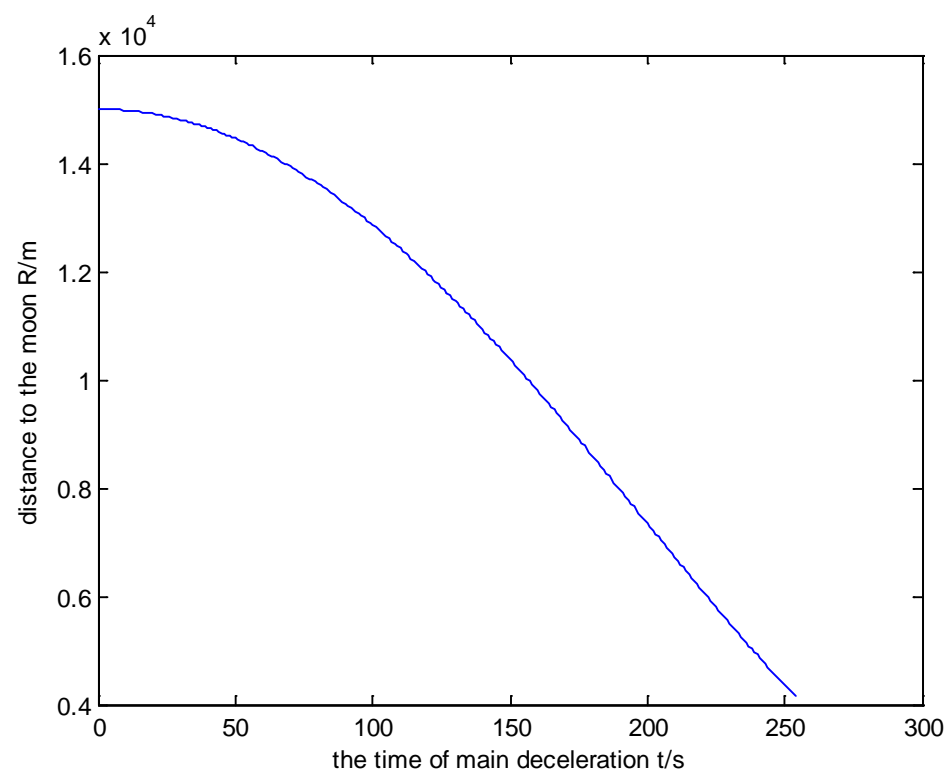

Figure 2 Curve of month pitch changes with time

Table 1 Result of main reduction stage and obstacle avoidance stage

\begin{tabular}{|c|c|c|c|c|c|c|}
\hline & & & \multicolumn{2}{|c|}{$\begin{array}{c}\text { beginning and end } \\
\text { velocity }\end{array}$} & \multicolumn{2}{|c|}{ Fuel consumption } \\
\cline { 4 - 7 } Stage & $\begin{array}{c}\text { Decline of } \\
\text { altitude }\end{array}$ & $\begin{array}{c}\text { Elapsed } \\
\text { time(s) }\end{array}$ & $\begin{array}{c}\text { Initial } \\
\text { velocity } \\
\text { (m/s) }\end{array}$ & $\begin{array}{c}\text { Final } \\
\text { velocity } \\
\mathrm{m} / \mathrm{s})\end{array}$ & Amount (kg) & $\begin{array}{c}\text { Proportion } \\
\text { of total } \\
\text { quality }\end{array}$ \\
\hline $\begin{array}{c}\text { Main reduction } \\
\text { stage }\end{array}$ & $\begin{array}{c}15000-3000 \\
\mathrm{~m}\end{array}$ & 412.42 & 1700 & 57 & 1052.04 & $43.84 \%$ \\
\hline $\begin{array}{c}\text { Coarse } \\
\text { obstacle } \\
\text { avoidance }\end{array}$ & $2400-100 \mathrm{~m}$ & 68.07 & 0 & 0 & 34.73 & $1.45 \%$ \\
\hline $\begin{array}{c}\text { Essence of } \\
\text { obstacle } \\
\text { avoidance }\end{array}$ & $100-30 \mathrm{~m}$ & 5.08 & 0 & 8.25 & 2.59 & $0.108 \%$ \\
\hline
\end{tabular}

It can be learnt from the result that elapsed time of reduction phase is $412.42 \mathrm{~s}$, which is shorter than actual soft landing's(the real time is 10 mins).And fuel consumption is $1052.04 \mathrm{~kg}$, only $43.8 \%$ to total mass, less than $50 \%$ to fuel consumption from the whole course of soft landing. Thus, it would reduce fuel consumption,while soft land immensely and relieve the weight of detector when the orbit control strategy is adopted.

\section{Conclusion}

In this paper, GA is put into use to design reduction phase of Chang'e no. 3 soft landing, whose ability to global searching is used to get orbit scheme. Texture analysis is also applied to handle lunar surface, which makes Chang'e no.3 recognize obstacles area fast and respond to it. In brief, orbit scheme, which makes fuel consumption minimum, takes full advantage of GA and texture analysis when designing.

\section{References}

[1] Wang Jie,Li Junfeng,Cuinai Gang,Liu Dun.Genetic Algorithm Optimization of Lunar Probe Soft-landing Trajectories[J].JTsinghua Univ(Sci\&Tech), 2003, 43(8):1056-1059.

[2] Jiang Xiaonan,Han Chengchan,Li Xiangzhi.Multi-Sensor Based Lunar Surface Hazards Fuzzy Detecting Method[J].The Computer Simulation, 2013, 30(4):100-102.Xiaonan,Jiang, Chenshan, 
Han, Xiangzhi,Li. Multisensor fuzzy recognition method for lunar surface barrier [J]. Computer Simulation,2013,30(4):100-102.

[3] Liu Lonigfei,Chen Yunhao,Li Jing.Texture Analysis Methods Used In Remotr Sensing Images[J].REMOTE SENSING TECHNOLOGY AND APPLICATION, 2003, 18(6):441-447. 